\title{
Mid-infrared Gas Sensing Using Graphene Plasmons Tuned by Reversible Chemical Doping
}

\author{
Nestor Jr. Bareza, Kavitha K. Gopalan, Rose Alani, Bruno Paulillo,* and Valerio Pruneri \\ Cite This: https://dx.doi.org/10.1021/acsphotonics.9b01714 \\ Read Online
}

ACCESS I

Lلll Metrics \& More

Article Recommendations

Supporting Information

ABSTRACT: Highly confined plasmon modes in nanostructured graphene can be used to detect tiny quantities of biological and gas molecules. In biosensing, a specific biomarker can be concentrated close to graphene, where the optical field is enhanced, by using an ad-hoc functional layer (e.g., antibodies). Inspired by this approach, in this paper we exploit the chemical and gas adsorption properties of an ultrathin polymer layer deposited on a nanostructured graphene surface to demonstrate a new gas sensing scheme. A proof-of-concept experiment using polyethylenimine
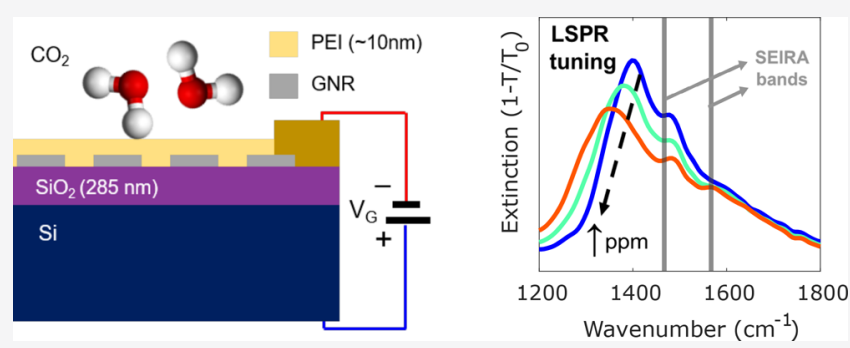
(PEI) that is chemically reactive to $\mathrm{CO}_{2}$ molecules is presented.

Upon $\mathrm{CO}_{2}$ adsorption, the sensor optical response changes because of PEI vibrational modes enhancement and shift in plasmon resonance, the latter related to polymer-induced doping of graphene. We show that the change in optical response is reversed during $\mathrm{CO}_{2}$ desorption. The demonstrated limit of detection (LOD) of $390 \mathrm{ppm}$ corresponds to the lowest value detectable in ambient atmosphere, which can be lowered by operating in vacuum. By using specific adsorption polymers, the proposed sensing scheme can be easily extended to other relevant gases, for example, volatile organic compounds.

KEYWORDS: graphene plasmonics, graphene sensor, gas sensor, SEIRA, chemical doping

G as sensing technology covers a wide range of applications such as air quality monitoring, ${ }^{1}$ hazard assessment, ${ }^{2}$ pipeline leakage detection, ${ }^{3}$ and breath diagnostics. ${ }^{4}$ More specifically, it is suitable for detecting volatile organic compounds (VOCs) and $\mathrm{CO}_{2}$ levels in domestic and work environments. Since an excess of $\mathrm{CO}_{2}$ is known to affect negatively human health and productivity, $\mathrm{CO}_{2}$ sensors can potentially become widespread components for integrated indoor air quality (IAQ) monitors, and smart ventilation systems. ${ }^{5,6}$

The gas sensors market is currently dominated by electrochemical technology that traditionally employs a metal oxide semiconductor as transducer element, where the chemical interaction with the target gas is converted into an electrical readout signal (e.g., resistance change). ${ }^{7}$ In recent years novel materials such as conducting polymers, ${ }^{8}$ metal nanoparticles, 9 and carbon nanomaterials (carbon nanotubes, graphene $)^{10}$ have enlarged the panorama of chemiresistive elements. In particular, single layer graphene field effect transistors (GFETs) with subnanoribbon channel allow high sensitive gas detection. ${ }^{11,12}$ Despite widespread use of chemiresistors in industry due to their low cost and possible miniaturization, they suffer from poor selectivity and large hysteresis. Also, their response degrades over time upon continuous exposure to gases. ${ }^{13}$

Optical gas sensors directly target the vibrational fingerprints of gas molecules in the mid-infrared (MIR) spectral range $(\lambda=$ $2.5-15 \mu \mathrm{m})$ offering high sensitivity, fast response, and large device lifetime. ${ }^{14,15}$ Nondispersive IR (NDIR) sensors are simple spectroscopic devices that measure how much MIR light of a specific wavelength is absorbed in a certain volume due to the presence of a gas. ${ }^{16}$ Compared to electrochemical sensors, NDIR sensors show neither hysteresis nor degradation over time, but their specificity is limited in the case of gas mixtures since the vibrations of different gas molecules (including water vapor) can overlap in frequency. ${ }^{17}$ Several optical techniques based on laser absorption spectroscopy (LAS) have been developed to detect gas traces with a subppb detection limit such as cavity-enhanced spectroscopy (CEAS), ${ }^{18}$ cavity ring-down spectroscopy (CRDS), ${ }^{19}$ and quartz-enhanced photoacoustic spectroscopy (QEPAS). ${ }^{20}$ These techniques are effective for critical industrial or military applications and for biomedical diagnostics. However, they are not suitable for large deployment and consumer applications due to the high cost of their components (e.g., MIR quantum cascade laser sources).

A way to overcome the weak interaction of IR light with gas molecules in a spectroscopic scheme is to enhance molecules-

Received: December 4, 2019

Published: March 18, 2020 
(a)

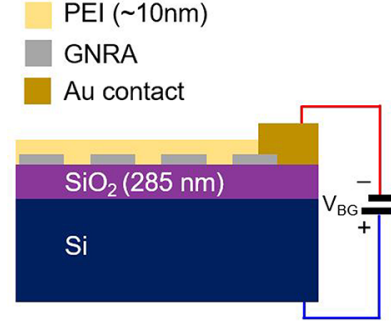

(b)

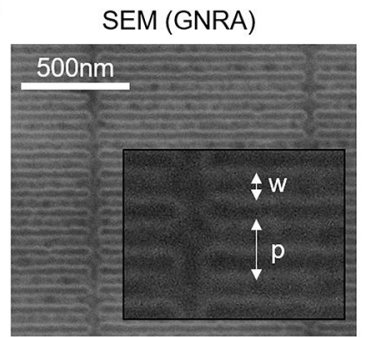

(c)

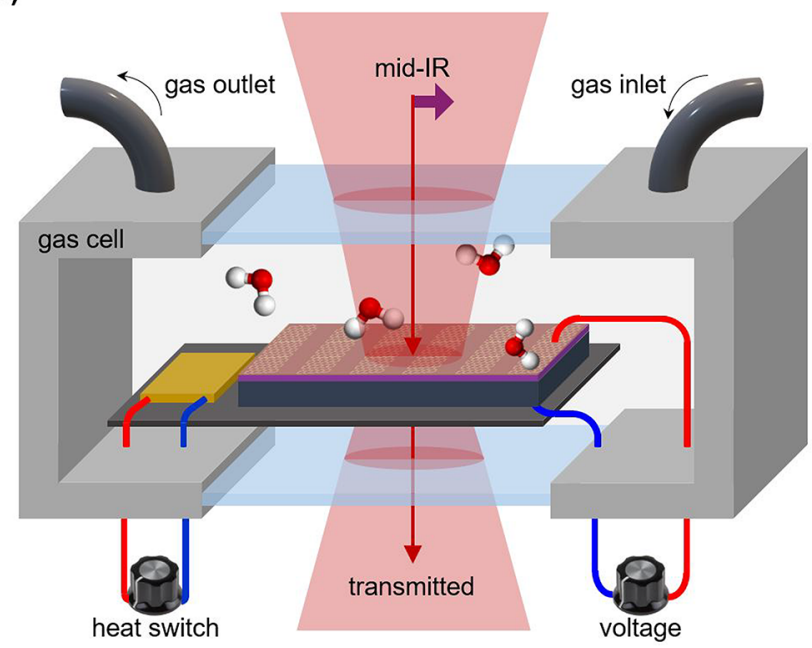

Figure 1. (a) Cross section of the sensor chip consisting of a GNRA on $\mathrm{SiO}_{2} / \mathrm{Si}$ substrate coated with an ultrathin PEI layer. A back-gate voltage is used for electrostatic tuning of graphene. (b) SEM micrograph of the GNRA with width $w=50 \mathrm{~nm}$ and period $p=90 \mathrm{~nm}$. (c) Schematic diagram of gas-sensing setup. The sensor is mounted on a heating stage inside a gas cell with IR-transparent windows. The optical reading is carried out with a FTIR microscope in the transmission mode.
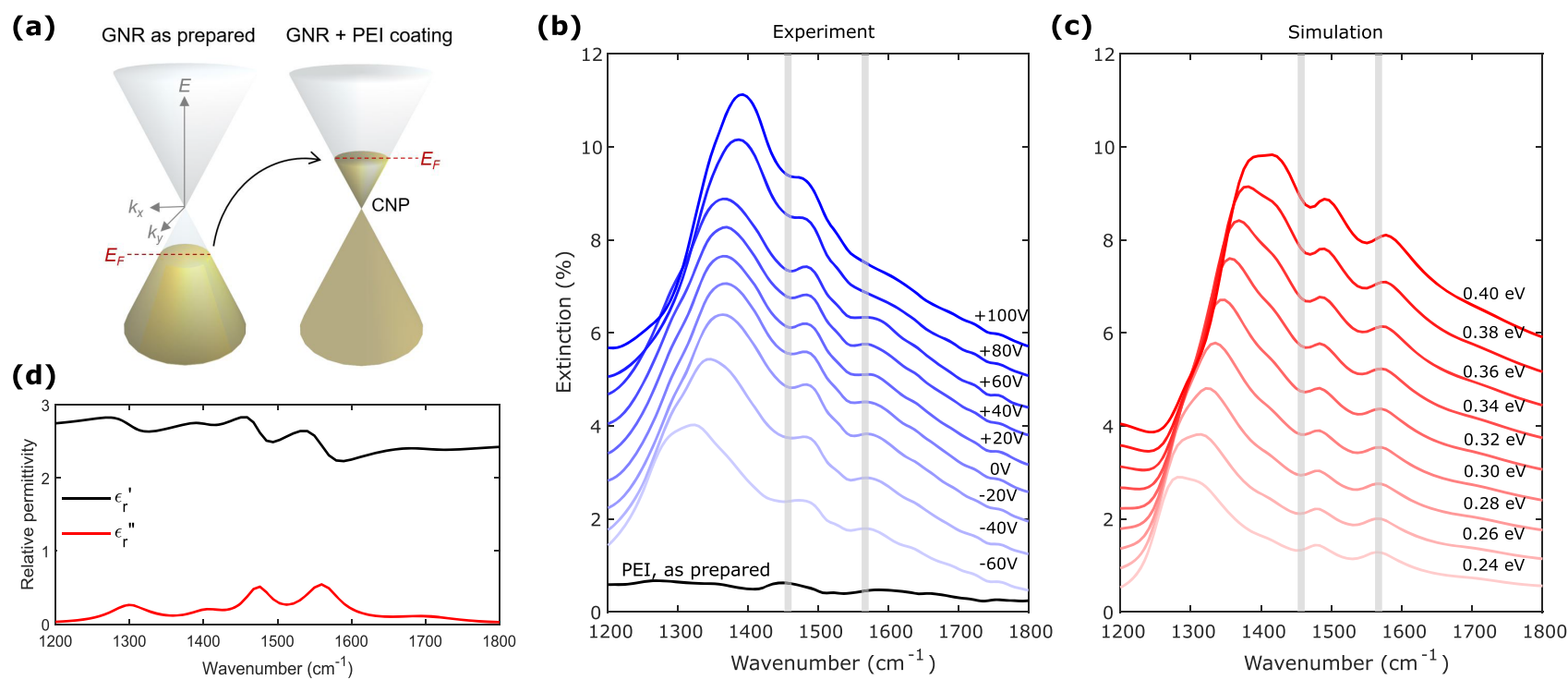

Figure 2. (a) Sketch of the graphene Dirac cone for the as-prepared GNR surfaces (p-doped) and GNR+PEI surfaces (n-doped). (b) Experimental extinction spectra of GNR functionalized with $10 \mathrm{~nm}$ PEI exposed in ambient condition at different $V_{\mathrm{BG}}$. (c) Simulated extinction spectra for $E_{\mathrm{F}}$ levels ranging from 0.24 to $0.40 \mathrm{eV}$. Gray lines indicate the prominent vibrational bands of PEI. Both experimental and simulated spectra are offset for clarity. (d) Relative permittivity $\epsilon=\epsilon_{\mathrm{r}}^{\prime}-i \epsilon_{\mathrm{r}}{ }^{\prime \prime}$ of ultrathin PEI extracted from experimental transmission data.

light interaction using highly confined surface modes in nanostructured metals, graphene (localized surface plasmon resonance, $\mathrm{LSPR}^{21,22}$ ) or dielectrics (surface phonon polaritons, $\left.\mathrm{SPhP}^{23-25}\right)$. In particular, graphene is emerging as a superior plasmonic material for surface-enhanced IR absorption (SEIRA) owing to its optical modes tunability via electrostatic gating ${ }^{26}$ or chemical doping. ${ }^{27}$ Moreover, the extreme field confinement enabled by graphene LSPR modes allows probing molecular vibrations in a very small volume close to the sensor surface $(d<15 \mathrm{~nm}) .^{28}$

In a recent work, graphene LSPR modes have been successfully used to identify $\mathrm{SO}_{2}, \mathrm{NO}_{2}$, and $\mathrm{N}_{2} \mathrm{O}$ gases in a SEIRA setup. ${ }^{29}$ However, the measured absorption signals were weak due to the small number of gas molecules physically adsorbing on the surface where the light-matter interaction takes place and the reported limit of detection (LOD) was above 800 ppm.

In this paper, we propose a radically different approach that consists in introducing an ultrathin functional layer with high specificity to selectively concentrate the gas molecules in proximity of the graphene-based SEIRA surface structure. Remarkably, a smart gas trapping polymer with ad-hoc functional groups produces chemisorption of specific gas molecules. ${ }^{30}$ This is similar to the role that antibodies have in optical biosensors to capture well selected biomarkers (SPR, SEIRA). ${ }^{28}$ As a proof-of-concept we used an ultrathin polyethylenimine (PEI) coating to selectively concentrate and detect $\mathrm{CO}_{2}$ molecules close to the graphene plasmonic nanostructures. ${ }^{30}$ In our approach, two coexisting transduction mechanisms contribute to sensing: (i) the change in strength 
(a)

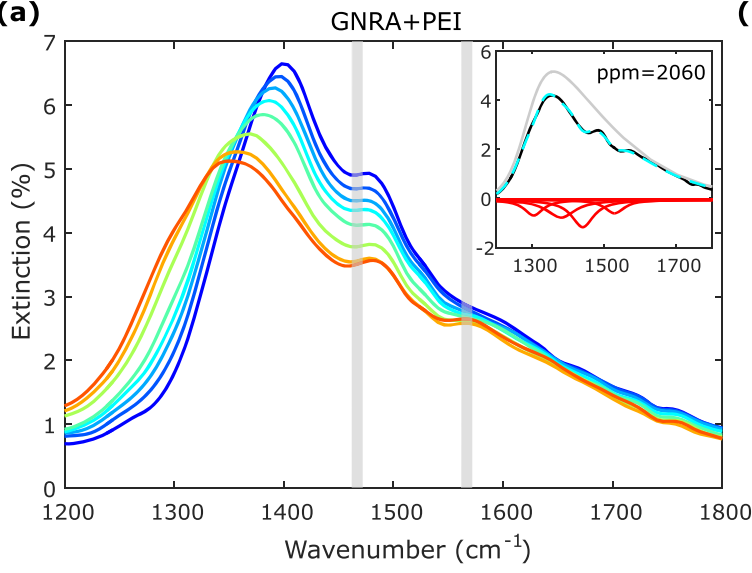

(b)

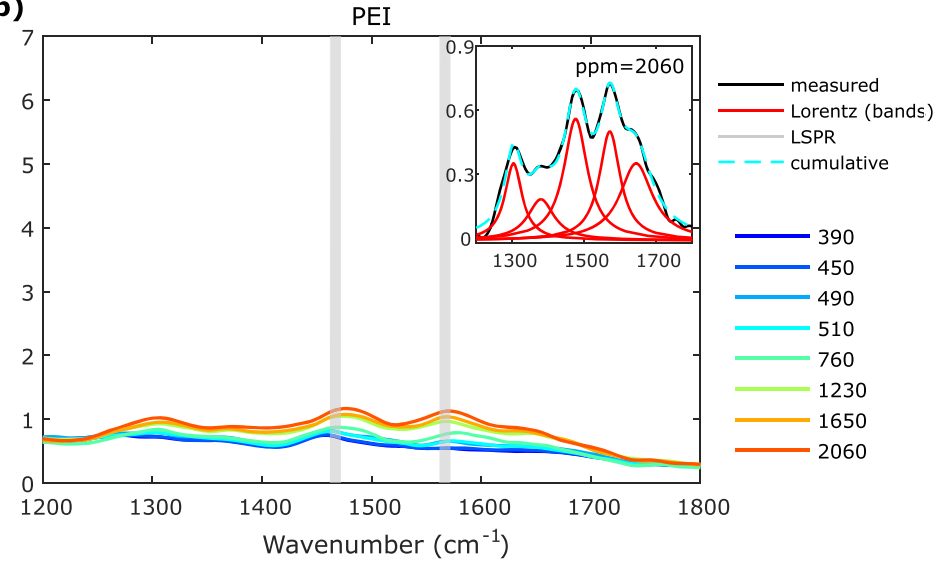

(c)

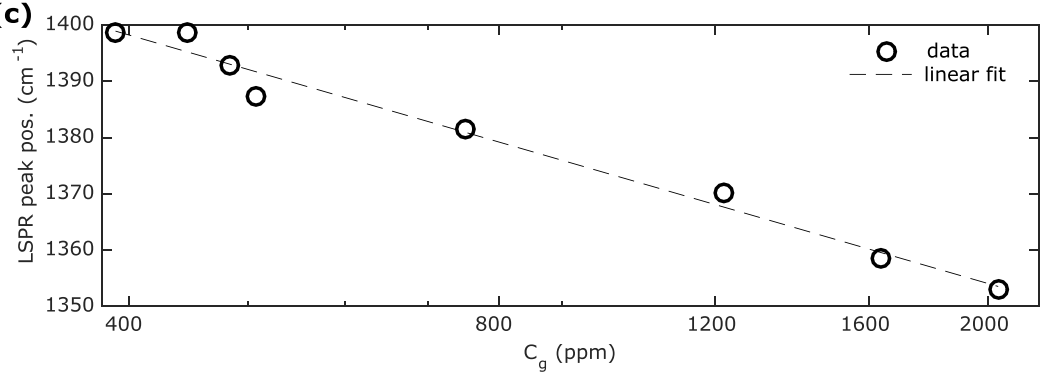

Figure 3. Extinction spectra of PEI film exposed under different ppm levels of $\mathrm{CO}_{2}$ (a) with and (b) without GNRA. Insets show the spectra at ppm $=2060$ with extraction of plasmonic and Lorentzian vibrational frequency peaks. (c) LSPR peak position as a function of gas concentration.

of vibrational bands (conventional SEIRA effect) of the PEI layer and (ii) the tuning of LSPR modes via graphene chemical doping assisted by PEI, both occurring as a result of $\mathrm{CO}_{2}$ molecules adsorption. Note that, compared to previous work, ${ }^{29}$ the use of PEI overcomes the limited molecular interaction of free graphene SEIRA structures with gases and produces in this way higher sensitivity. An LOD of $390 \mathrm{ppm}$ was measured in these initial experiments. A lower LOD could not be achieved in our setup because we were limited by the fact that we operate in an ambient atmosphere.

The proposed hybrid sensor is depicted in Figure 1a. It consists of a graphene nanoribbon (GNR) array fabricated on a conventional $\mathrm{Si} / \mathrm{SiO}_{2}$ substrate and coated with an ultrathin PEI film (see GNR and ultrathin PEI fabrication in Methods). The SEM micrograph (Figure $1 b$ ) shows the typical graphene nanoribbon array (GNRA) geometry to operate around 1300$1600 \mathrm{~cm}^{-1}$ (where PEI vibrational bands are located ${ }^{30}$ ): GNR width is $50 \mathrm{~nm}$ and period is $90 \mathrm{~nm}$. The GNRs in the array are connected to each other for allowing electrostatic gating of the entire surface. Since the evanescent field decay length of graphene LSPR modes is $\sim 15 \mathrm{~nm}^{28}$ a PEI film of thickness $\sim 10 \mathrm{~nm}$ (film characterizations in SI) is chosen to maximize the spatial overlap with the GNR plasmonic field. In Figure 1c the schematic diagram of the MIR gas characterization setup is shown. The sensor is mounted inside a gas cell with IRtransparent windows. A heating stage allows desorbing gas from the surface to regenerate the PEI film. A Fourier transform IR (FTIR) microscope in transmission mode is used for optical excitation/detection using light polarized perpendicular to GNRs (additional details in Methods).

The optical characterization of the sensing surface is presented in Figure 2. Note that PEI is known to act as ndopant for graphene, shifting the position of the charge neutrality point $(\mathrm{CNP})$ from positive gate voltage $\left(V_{\mathrm{CNP}}>0\right.$, i.e., Fermi level in the valence band, typical of as-prepared graphene devices on $\left.\mathrm{Si} / \mathrm{SiO}_{2}{ }^{31,32}\right)$ to negative voltages $\left(V_{\mathrm{CNP}}<\right.$ 0 , i.e., Fermi level in the conduction band ${ }^{33,34}$ ), as sketched in Figure 2a. As a consequence, to blueshift and enhance graphene plasmons in PEI-coated graphene, one has to bias the device toward positive $V_{\mathrm{BG}}$ (i.e., higher n-doping), unlike the case of graphene alone where plasmons are enhanced going toward negative $V_{\mathrm{BG}}$ (i.e., higher $\mathrm{p}$-doping)..$^{31,32,35}$ This is experimentally observed in Figure $2 b$, where the extinction spectra of the GNR surface covered by a $\sim 10 \mathrm{~nm}$ PEI layer as a function of $V_{\mathrm{BG}}$ are shown. We observe that the LSPR mode is strongly visible even at $V_{\mathrm{BG}}=-60 \mathrm{~V}$ bias, indicating that ultrathin PEI coating induces locally a substantial n-type chemical doping. For comparison, the extinction spectra as a function of $V_{\mathrm{BG}}$ for the same GNR array before PEI functionalization are reported in Figure S1 of Supporting Information. The initial position of the Dirac point for the asprepared GNR surface can be located around $V_{\mathrm{CNP}} \approx+20 \mathrm{~V}$. To estimate instead the initial position of the Fermi level for the GNR+PEI surface, it is useful to compare the experimental spectra of Figure $2 b$ with the simulated extinction spectra of the structure for various graphene Fermi energies $E_{\mathrm{F}}$. In particular, the experimental LSPR spectrum at $V_{\mathrm{BG}}=0$ is comparable to the simulated spectra with $E_{\mathrm{F}} \approx 0.32 \mathrm{eV}$, meaning that the Dirac point can be roughly located around $V_{\mathrm{CNP}} \approx-80 \mathrm{~V}$ (calculation of the Dirac curves for GNR and GNR+PEI in the SI). In addition, the coupling between graphene LSPR mode and the molecular vibrations of PEI is apparent in Figure $2 \mathrm{c}$ where two local dips in the plasmonic resonance are visible at around 1475 and $1565 \mathrm{~cm}^{-1}$ (more details in the SI). These two weak vibrational bands are enhanced by the plasmonic field, as expected in a SEIRA sensing scheme. For comparison, the extinction spectrum of the ultrathin PEI film alone is reported (black curve in Figure 
$2 \mathrm{~b})$. In the simulations, we model the optical properties of PEI with a complex permittivity (Figure $2 \mathrm{~d}$ ) extracted by fitting experimental transmission data of PEI films on a transparent IR substrate (more information in the SI). Using this model, we are able to simulate with good agreement the SEIRA effect observed experimentally.

The spectral response of the sensor under $\mathrm{CO}_{2}$ exposure is shown in Figure 3a. The $\mathrm{CO}_{2}$ levels in the gas cell (expressed in ppm) are monitored using a commercial $\mathrm{CO}_{2}$ sensor. The heating stage installed on the mounting allows releasing $\mathrm{CO}_{2}$ adsorbed in the PEI layer to regenerate the sensor. We fixed $V_{\mathrm{BG}}=+100 \mathrm{~V}$ to allow maximum overlap between the LSPR mode and PEI vibrational bands and we changed the $\mathrm{CO}_{2}$ concentration from the initial minimum of $390 \mathrm{ppm}$ (close to the atmospheric value ${ }^{36}$ ) to a maximum of $\sim 2000 \mathrm{ppm}$. Note that the lowest value for the $\mathrm{CO}_{2}$ detection in our work is limited to the atmospheric concentration since our setup is not hermetically sealed and atmospheric $\mathrm{CO}_{2}$ leaks into the gas cell. This leads to an LOD larger than the value one would get if the measurements were performed in an airtight setup. The experiments show that when the $\mathrm{CO}_{2}$ level is increased, the LSPR continuously redshifts and its intensity reduces, that is consistent with a carrier decrease in the graphene (or reduction of graphene doping). In contrast, the spectral response of a GNR array without PEI coating shows no significant changes upon $\mathrm{CO}_{2}$ exposure (Figure $\mathrm{S} 5$ in Supporting Information).

A representative deconvolution of the measured spectrum for a certain $\mathrm{CO}_{2}$ level is reported in the figure inset to distinguish the LSPR and vibrational mode contributions (details of fit in SI). As a comparison, in Figure $3 \mathrm{~b}$ the behavior response of PEI alone on an unpatterned graphene zone of the same chip is shown. The growth in intensity of the vibrational bands upon $\mathrm{CO}_{2}$ exposure is weaker than before since no surface-enhancement effect is present and no appreciable spectral shift is observed. The figure inset shows the deconvolution of the PEI bands.

Notably, the LSPR tuning via chemical doping is a new sensing mechanism, as it directly relates the LSPR peak shift induced by the change in doping with the gas concentration. It could potentially lead to improved performance since reading a peak wavelength shift is simpler than monitoring the evolution of the SEIRA response.

In the following, we elucidate the relation between $\mathrm{CO}_{2}$ concentration and the change in graphene $E_{\mathrm{F}}$, equivalent to the change in chemical doping. From the spectra in Figure $2 b, c$, we estimate that the total doping of the surface biased at $V_{\mathrm{BG}}=$ $+100 \mathrm{~V}$ and exposed to atmospheric $\mathrm{CO}_{2}(390 \mathrm{ppm})$ is equivalent to $E_{\mathrm{F}}^{\mathrm{atm},+100 \mathrm{~V}} \approx 0.4 \mathrm{eV}$, which produces the initial spectrum in Figure 3a (blue curve). If we limit our analysis to the zone of linear response (up to 2000 ppm, see next Figure 4 ), the change in chemical doping due to $\mathrm{CO}_{2}$ exposure can be written as

$$
\frac{\Delta E_{\mathrm{F}}}{\Delta \log \left(C_{\mathrm{gas}}\right)}=\frac{\Delta E_{\mathrm{F}}}{\Delta k_{\mathrm{LSPR}}} \cdot \frac{\Delta k_{\mathrm{LSPR}}}{\Delta \log \left(C_{\mathrm{gas}}\right)}
$$

The dispersion of LSPR peak position $k_{\mathrm{LSPR}}$ versus the gas concentration $\log \left(C_{\text {gas }}\right)$ is shown in Figure $3 \mathrm{c}$. By linear fitting the experimental data, we obtain a slope $\frac{\Delta k_{\mathrm{LSPR}}}{\Delta \log \left(C_{\mathrm{gas}}\right)}=-63 \mathrm{~cm}^{-1} / \mathrm{ppm}$. The dependence $k_{\mathrm{LSPR}}$ versus the $E_{\mathrm{F}}$ can be derived analytically or from simulations (see

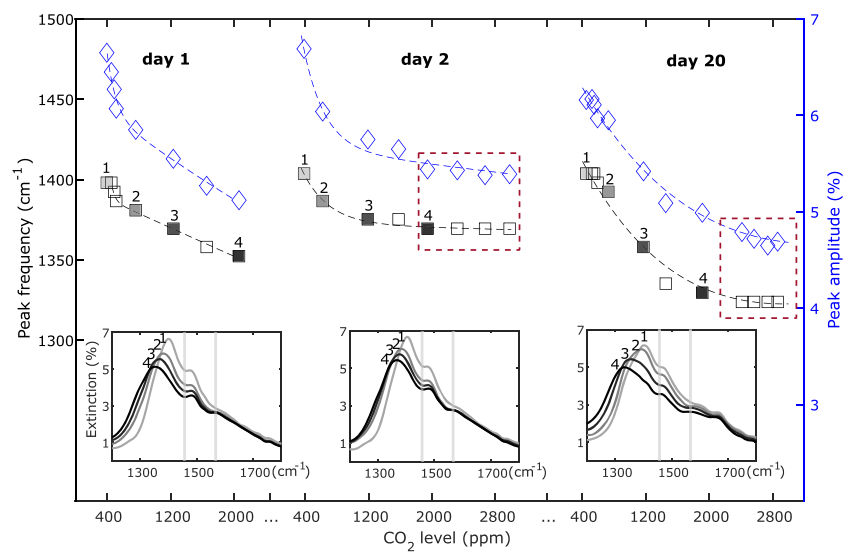

Figure 4. Peak frequency and amplitude for three measurement cycles taken at different days. The red dashed boxes show the saturation region. Selected spectra for each cycle are plotted below and identified with numbers.

SI), yielding $\frac{\Delta k_{\mathrm{LSPR}}}{\Delta E_{\mathrm{F}}}=1216 \mathrm{~cm}^{-1} / \mathrm{eV}$. We use eq 1 to calculate the relative change in graphene $E_{\mathrm{F}}$ for a given increase in $\mathrm{CO}_{2}$ concentration from the atmospheric value. The expected decrease in $E_{\mathrm{F}}$ for high $\mathrm{CO}_{2}$ level $\left(\Delta C_{\mathrm{g}} \approx 1000 \mathrm{ppm}\right)$ is around $\Delta E_{\mathrm{F}} \approx 0.1 \mathrm{eV}$, which yields $E_{\mathrm{F}}^{1000 \mathrm{ppm},+100 \mathrm{~V}} \approx 0.3 \mathrm{eV}$, compatible to a redshift in the LSPR peak position corresponding to the one shown in the simulations of Figure $2 \mathrm{c}$.

The sensor response to $\mathrm{CO}_{2} \mathrm{ppm}$ variations have been recorded for three cycles of measurements. The LSPR peak position and intensity are plotted in Figure 4 and representative spectra per cycle are displayed in the corresponding inset. The PEI is regenerated by heating the device at $95{ }^{\circ} \mathrm{C}$ for $1.5 \mathrm{~min}$ to thermally desorb $\mathrm{CO}_{2}$. Upon exposure to $\mathrm{CO}_{2}$, we observe the same behavior previously described: the LSPR mode redshifts and decreases in intensity for higher ppm for all cycles. The response is linear in the range $390-2000 \mathrm{ppm}$, while a saturation behavior is observed for higher ppm. The latter is probably associated with the low thickness of the PEI film. Thicker films would enlarge the dynamic range by providing an initial higher doping. Note that the reported dynamic range is already relevant for applications as $1000 \mathrm{ppm}$ of $\mathrm{CO}_{2}$ is classified as harmful and requires ventilation. $^{37}$ Repeatability in cyclic measurements can be improved by employing a hermetically sealed gas cell to avoid exposing the surface to a changing atmosphere that alters graphene doping. Also, we note the presence of an extra PEI vibrational mode at $1684 \mathrm{~cm}^{-1}$ appearing in cycle 3 (20th day), which is also observed when looking at a region of PEI without GNR (cycle measurements in the SI). This can be associated with slow urea formation over time, ${ }^{38}$ which may degrade the chemisorptive layer and affect repeatability and device lifetime. This issue can be improved by stabilizing the PEI coating formulation (e.g., adding cross-linkers to the PEI solution). ${ }^{39}$ Moreover, we have evaluated that the ultrathin PEI coatings that we have used react to change in $\mathrm{CO}_{2}$ concentration in less than $1 \mathrm{~min}$, in agreement with previous work that reported $\approx 2$ min for a $300 \mathrm{~nm}$ layer. ${ }^{30}$

In conclusion, we have demonstrated a novel platform for mid-infrared gas sensing based on a nanostructured graphene surface coated with an ultrathin gas adsorbing polymer. Sensing of $\mathrm{CO}_{2}$ is attributed to two separate transduction mechanisms. On the one hand, captured $\mathrm{CO}_{2}$ changes the 
polymer vibrational bands intensity through the well-known SEIRA effect. On the other hand, for the first time, we have demonstrated that the polymer-induced graphene doping upon gas exposure provides an efficient sensing mechanism since $\mathrm{CO}_{2}$ levels can be monitored through the changes in the LSPR response. This proof-of-concept demonstration with $\mathrm{CO}_{2}$ can be readily extended to the detection of more gas species using the same polymer adsorber (e.g., $\mathrm{NH}_{3}$ and acetone ${ }^{40}$ ) or different conductive polymers. ${ }^{4}$ Furthermore, quantitative information in the presence of a gas mixture could be obtained by monitoring both the doping-induced plasmonic shift and the vibrational band intensities of the polymer and, where available, the intrinsic gas vibrational fingerprints (see example of acetone versus $\mathrm{CO}_{2}$ sensing with PEI in ${ }^{30}$ ). Our results pave the way toward implementing miniaturized optical environmental sensors with ad hoc functional layers for various environmental pollutants (i.e., VOCs). Moreover, further study of polymer-assisted graphene chemical doping can result in the realization of gate-free SEIRA sensing surfaces.

\section{METHODS}

EBL Fabrication of GNR. Double-side polished silicon with a $285 \mathrm{~nm}$ native $\mathrm{SiO}_{2}$ layer on one side is used as the substrate. Graphene grown by chemical vapor deposition on a copper catalyst (from Graphenea Inc.) was wet-transferred to the $\mathrm{Si} / \mathrm{SiO}_{2}$ substrate. A $40 \mathrm{~nm}$ thick layer of electron beam resist (ZEP520A by Zeon) was spin-coated on the chip. Subsequently, GNR arrays with different dimensions were exposed using a $50 \mathrm{keV}$ electron beam lithography system (CRESTEC). After developing, the exposed graphene regions are removed by reactive ion etching with oxygen/argon plasma at $10 \mathrm{~W}$ for $1 \mathrm{~min}$. The resist is removed with acetone and subsequently rinsed with IPA.

Ultrathin PEI Preparation. Branched PEI $\left(M_{\mathrm{w}} \sim 25000\right.$ from Sigma-Aldrich) is diluted in ethanol by magnetic stirring to obtain a 0.22 wt $\%$ solution. The chips are spin-coated with PEI solution at $5000 \mathrm{rpm}$ and baked at $100{ }^{\circ} \mathrm{C}$ for $2 \mathrm{~min}$. The PEI coating characterizations (thickness, surface morphology and FTIR) are detailed in the SI.

Optical Measurement. Fourier transform IR spectrometer (Bruker Tensor II) equipped with an IR microscope (Bruker Hyperion 2000) is used to collect the transmission spectra. The beam is focused onto the chip surface with a Cassegrain objective of NA $=0.4$ and $15 \times$ magnification. IR light is polarized perpendicular to GNR orientation. The transmitted IR light is collected by liquid nitrogen-cooled mercurycadmium-tellurium detector. Subsequent measurements were taken on regions with (signal, $T$ ) and without (background, $\left.T_{0}\right)$ GNR+PEI. Extinction is defined as $1-T / T_{0}$. The measurement of each spectrum is collected for $1 \mathrm{~min}$.

Finite Element Simulation of GNR. Electromagnetic simulations in Figure $2 \mathrm{c}$ are performed using a Finite Elements Method commercial software (Comsol). A GNR array unit cell is simulated using periodic boundary conditions. Graphene is modeled as a two-dimensional surface with complex Drude-like conductivity $\sigma_{\text {intra }}=\frac{e^{2}}{\pi \hbar^{2}(\gamma-i \omega)}\left[E_{\mathrm{F}}+2 k_{\mathrm{B}} T \ln \left(1+e^{-E_{\mathrm{F}} / k_{\mathrm{B}} T}\right)\right]$ accounting for intraband processes. ${ }^{42} \mathrm{SiO}_{2}$ permittivity dispersion is taken from Kitamura. ${ }^{43}$ PEI complex permittivity (Figure 2d) is derived by fitting experimental transmission data, as described in the SI. In the simulations, the graphene damping is set to $\gamma=6.7 \times 10^{13} \mathrm{~s}^{-1}$ to compare with the experimental LSPR bandwidth.

\section{ASSOCIATED CONTENT}

\section{(3) Supporting Information}

The Supporting Information is available free of charge at https://pubs.acs.org/doi/10.1021/acsphotonics.9b01714.

(S1) The extinction response of the as-prepared GNR surface in varying electrostatic voltage back-gating. (S2) Interaction of PEI film with $\mathrm{CO}_{2}$ and their vibrational fingerprints. (S3) Ultrathin PEI film characterizations such as thickness, surface morphology and desorption behavior. (S4) Fit of PEI permittivity model obtained from experimental transmission spectrum. (S5) Sensing of bare GNR with varying $\mathrm{CO}_{2}$ concentration. (S6) Deconvolution of LSPR and PEI vibrational frequency peaks. (S7) Cyclic $\mathrm{CO}_{2}$ sensing of ultrathin PEI film without GNR. (S8) GNR LSPR peak position as a function of Fermi energy (PDF)

\section{AUTHOR INFORMATION}

\section{Corresponding Author}

Bruno Paulillo - ICFO-Institut de Ciencies Fotoniques, The Barcelona Institute of Science and Technology, 08860 Castelldefels, Barcelona, Spain; 이이.org/0000-0002-66750141; Email: bruno.paulillo@icfo.eu

\section{Authors}

Nestor Jr. Bareza - ICFO-Institut de Ciencies Fotoniques, The Barcelona Institute of Science and Technology, 08860 Castelldefels, Barcelona, Spain

Kavitha K. Gopalan - ICFO-Institut de Ciencies Fotoniques, The Barcelona Institute of Science and Technology, 08860 Castelldefels, Barcelona, Spain; (1) orcid.org/0000-0002-25703031

Rose Alani - ICFO-Institut de Ciencies Fotoniques, The Barcelona Institute of Science and Technology, 08860 Castelldefels, Barcelona, Spain

Valerio Pruneri - ICFO-Institut de Ciencies Fotoniques, The Barcelona Institute of Science and Technology, 08860 Castelldefels, Barcelona, Spain; ICREA-Institució Catalana de Recerca i Estudis Avançats, 08010 Barcelona, Spain

Complete contact information is available at: https://pubs.acs.org/10.1021/acsphotonics.9b01714

\section{Funding}

We acknowledge financial support from the Spanish Ministry of Economy and Competitiveness through the "Severo Ochoa" Programme for Centres of Excellence in R\&D (SEV-20150522) and OPTO-SCREEN (TEC2016-75080-R), from Fundació Privada Cellex, from Fundació Mir-Puig, from Generalitat de Catalunya through the CERCA Program. The research leading to these results has received funding from the H2020 Programme under Grant Agreement No. 785219 Graphene Flagship. This project has received funding from the European Union's Horizon 2020 research and innovation program under the Marie Skłodowska-Curie Grant Agreement No. 665884. This project has received funding from the European Union's Horizon 2020 research and innovation program under the Marie Skłodowska-Curie Grant Agreement No. 754510. R.A. acknowledges funding from the Mujeres por Africa Foundation.

Notes

The authors declare no competing financial interest. 


\section{ACKNOWLEDGMENTS}

We thank Tetiana Slipchenko, Luis M. Moreno, and Raivo Jaaniso for useful discussions. We thank Daniel Martinez for help with AFM measurements.

\section{REFERENCES}

(1) Fine, G. F.; Cavanagh, L. M.; Afonja, A.; Binions, R. Metal Oxide Semi-Conductor Gas Sensors in Environmental Monitoring. Sensors 2010, 10 (6), 5469-5502.

(2) Kumar, A.; Kingson, T. M. G.; Verma, R.P.; Kumar, A.; Mandal, R.; Dutta, S.; Chaulya, S. K.; Prasad, G. M. Application of Gas Monitoring Sensors in Underground Coal Mines and Hazardous Areas. Int. J. Comput. Technol. Electron. Eng. 2013, 3 (3), 9-23.

(3) Murvay, P. S.; Silea, I. A Survey on Gas Leak Detection and Localization Techniques. J. Loss Prev. Process Ind. 2012, 25, 966.

(4) Di Natale, C.; Paolesse, R.; Martinelli, E.; Capuano, R. SolidState Gas Sensors for Breath Analysis: A Review. Anal. Chim. Acta 2014, 824, 1.

(5) Kwon, J.; Ahn, G.; Kim, G.; Kim, J. C.; Kim, H. A Study on NDIR-Based CO2 Sensor to Apply Remote Air Quality Monitoring System. ICCAS-SICE 2009 - ICROS-SICE International Joint Conference 2009, Proceedings; IEEE, 2009.

(6) Pitarma, R.; Marques, G.; Ferreira, B. R. Monitoring Indoor Air Quality for Enhanced Occupational Health. J. Med. Syst. 2017, na DOI: $10.1007 / \mathrm{s} 10916-016-0667-2$.

(7) Jaaniso, R.; Tan, O. K. Semiconductor Gas Sensors 2013, na.

(8) Janata, J.; Josowicz, M. Conducting Polymers in Electronic Chemical Sensors. Nat. Mater. 2003, 2, 19.

(9) Riu, J.; Maroto, A.; Rius, F. X. Nanosensors in Environmental Analysis. Talanta 2006, 69 (2 SPEC. ISS.), 288-301.

(10) Joshi, N.; Hayasaka, T.; Liu, Y.; Liu, H.; Oliveira, O. N.; Lin, L. A Review on Chemiresistive Room Temperature Gas Sensors Based on Metal Oxide Nanostructures, Graphene and 2D Transition Metal Dichalcogenides. Microchim. Acta 2018, 185 (4), na DOI: 10.1007/ s00604-018-2750-5.

(11) Wang, X.; Ouyang, Y.; Li, X.; Wang, H.; Guo, J.; Dai, H. RoomTemperature All-Semiconducting Sub-10-Nm Graphene Nanoribbon Field-Effect Transistors. Phys. Rev. Lett. 2008, na DOI: 10.1103/ PhysRevLett.100.206803.

(12) Yuan, W.; Shi, G. Graphene-Based Gas Sensors. J. Mater. Chem. A 2013, 1 (35), 10078-10091.

(13) Khan, M. A. H.; Rao, M. V.; Li, Q. Recent Advances in Electrochemical Sensors for Detecting Toxic Gases: NO2, SO2 and H2S. Sensors 2019, 19, 905.

(14) Hodgkinson, J.; Tatam, R. P. Optical Gas Sensing: A Review. Meas. Sci. Technol. 2013, 24, 012004.

(15) Bogue, R. Detecting Gases with Light: A Review of Optical Gas Sensor Technologies. Sens. Rev. 2015, 35, 133.

(16) Wong, J. Y. NDIR Gas Sensor. U.S. Patent US5747808A, 1998.

(17) Stolberg-Rohr, T.; Buchner, R.; Clausen, S.; Jensen, J. M.; Skouboe, A.; Hawkins, G.; Hansen, R. S. In Optics Humidity Compensation in NDIR Exhaust Gas Measurements of NO2. Optical Sensors 2014, na.

(18) Wang, H.; Chen, J.; Lu, K. Development of a Portable CavityEnhanced Absorption Spectrometer for the Measurement of Ambient NO3 and N2O5: Experimental Setup, Lab Characterizations, and Field Applications in a Polluted Urban Environment. Atmos. Meas. Tech. 2017, 10, 1465

(19) Brown, S. S.; Stark, H.; Ravishankara, A. R. Cavity Ring-down Spectroscopy for Atmospheric Trace Gas Detection: Application to the Nitrate Radical (NO3). Appl. Phys. B: Lasers Opt. 2002, 75, 173.

(20) Patimisco, P.; Scamarcio, G.; Tittel, F. K.; Spagnolo, V. QuartzEnhanced Photoacoustic Spectroscopy: A Review. Sensors 2014, 14 (4), 6165-6206.

(21) Chong, X.; Zhang, Y.; Li, E.; Kim, K. J.; Ohodnicki, P. R.; Chang, C. H.; Wang, A. X. Surface-Enhanced Infrared Absorption: Pushing the Frontier for On-Chip Gas Sensing. ACS Sensors 2018, 3, 230.
(22) Koppens, F. H. L.; Chang, D. E.; García de Abajo, F. J. Nano Lett. 2011, 11 (8), 3370-3377.

(23) Chang, Y.; Hasan, D.; Dong, B.; Wei, J.; Ma, Y.; Zhou, G.; Ang, K. W.; Lee, C. All-Dielectric Surface-Enhanced Infrared AbsorptionBased Gas Sensor Using Guided Resonance. ACS Appl. Mater. Interfaces 2018, 10, 38272.

(24) Autore, M.; Li, P.; Dolado, I.; Alfaro-Mozaz, F. J.; Esteban, R.; Atxabal, A.; Casanova, F.; Hueso, L. E.; Alonso-González, P.; Aizpurua, J.; et al. Boron Nitride Nanoresonators for PhononEnhanced Molecular Vibrational Spectroscopy at the Strong Coupling Limit. Light: Sci. Appl. 2018, 7, 17172.

(25) Tittl, A.; Leitis, A.; Liu, M.; Yesilkoy, F.; Choi, D. Y.; Neshev, D. N.; Kivshar, Y. S.; Altug, H. Imaging-Based Molecular Barcoding with Pixelated Dielectric Metasurfaces. Science 2018, 360, 1105.

(26) Ju, L.; Geng, B.; Horng, J.; Girit, C.; Martin, M.; Hao, Z.; Bechtel, H. A.; Liang, X.; Zettl, A.; Shen, Y. R.; Wang, F. Graphene Plasmonics for Tunable Terahertz Metamaterials. Nat. Nanotechnol. 2011, 6, 630.

(27) Liu, H.; Liu, Y.; Zhu, D. Chemical Doping of Graphene. J. Mater. Chem. 2011, 21, 3335.

(28) Rodrigo, D.; Limaj, O.; Janner, D.; Etezadi, D.; García De Abajo, F. J.; Pruneri, V.; Altug, H. Mid-Infrared Plasmonic Biosensing with Graphene. Science (Washington, DC, U. S.) 2015, 349 (6244), $165-168$.

(29) Hu, H.; Yang, X.; Guo, X.; Khaliji, K.; Biswas, S. R.; García de Abajo, F. J.; Low, T.; Sun, Z.; Dai, Q. Gas Identification with Graphene Plasmons. Nat. Commun. 2019, 10 (1), 1-7.

(30) Hasan, D.; Lee, C. Hybrid Metamaterial Absorber Platform for Sensing of CO2 Gas at Mid-IR. Adv. Sci. 2018, 5 (5), 1700581.

(31) Rodrigo, D.; Tittl, A.; Limaj, O.; de Abajo, F. J. G.; Pruneri, V.; Altug, H. Double-Layer Graphene for Enhanced Tunable Infrared Plasmonics. Light: Sci. Appl. 2017, 6 (6), No. e16277.

(32) Brar, V. W.; Jang, M. S.; Sherrott, M.; Lopez, J. J.; Atwater, H. A. Highly Confined Tunable Mid-Infrared Plasmonics in Graphene Nanoresonators. Nano Lett. 2013, 13 (6), 2541-2547.

(33) Farmer, D. B.; Golizadeh-Mojarad, R.; Perebeinos, V.; Lin, Y.M.; Tulevski, G. S.; Tsang, J. C.; Avouris, P. Chemical Doping and Electron-Hole Conduction Asymmetry in Graphene Devices. Nano Lett. 2009, 9 (1), 388-392.

(34) Kim, S. Y.; Hwang, J.; Kim, Y. J.; Hwang, H. J.; Son, M.; Revannath, N.; Ham, M. H.; Cho, K.; Lee, B. H. Threshold Voltage Modulation of a Graphene-ZnO Barristor Using a Polymer Doping Process. Adv. Electron. Mater. 2019, 5 (7), 1800805.

(35) Gopalan, K. K.; Paulillo, B.; Mackenzie, D. M. A.; Rodrigo, D.; Bareza, N.; Whelan, P. R.; Shivayogimath, A.; Pruneri, V. Scalable and Tunable Periodic Graphene Nanohole Arrays for Mid-Infrared Plasmonics. Nano Lett. 2018, 18 (9), 5913-5918.

(36) Daily $\mathrm{CO}_{2}$. https://www.co2.earth/daily-co2.

(37) Mumma, S. a. Transient Occupancy Ventilation By Monitoring $\mathrm{CO}_{2}$. IAQ Appl. 2004, 21-23.

(38) Jiang, L.; Gao, L. Carbon Nanotubes-Magnetite Nanocomposites from Solvothermal Processes: Formation, Characterization, and Enhanced Electrical Properties. Chem. Mater. 2003, 15, 2848

(39) Zhang, S.; Kang, P.; Ubnoske, S.; Brennaman, M. K.; Song, N.; House, R. L.; Glass, J. T.; Meyer, T. J. Polyethylenimine-Enhanced Electrocatalytic Reduction of $\mathrm{CO} 2$ to Formate at Nitrogen-Doped Carbon Nanomaterials. J. Am. Chem. Soc. 2014, 136, 7845.

(40) Hine, J.; Sinha, A. Catalysis of.Alpha.-Hydrogen Exchange. Part 24. Bifunctional Catalysis of the Dedeuteration of Methoxyacetone1,1,3,3,3-D5. J. Org. Chem. 1984, 49, 2186-2190.

(41) Kumar, D.; Sharma, R. C. Advances in Conductive Polymers. Eur. Polym. J. 1998, 34, 1053.

(42) Falkovsky, L. A. Optical properties of graphene. J. Phys.: Conf. Ser. 2008, 129 (1), 012004.

(43) Kitamura, R.; Pilon, L.; Jonasz, M. Optical Constants of Silica Glass from Extreme Ultraviolet to Far Infrared at near Room Temperature. Appl. Opt. 2007, 46 (33), 8118 